\title{
Symmetries of general non-Markovian Gaussian diffusive unravelings
}

\author{
Adrián A. Budini \\ Consejo Nacional de Investigaciones Científicas y Técnicas (CONICET), \\ Centro Atómico Bariloche, Avenida E. Bustillo Km 9.5, (8400) Bariloche, \\ Argentina, and Universidad Tecnológica Nacional (UTN-FRBA), \\ Fanny Newbery 111, (8400) Bariloche, Argentina
}

(Dated: May 10, 2018)

\begin{abstract}
By using a condition of average trace preservation we re-derive a general class of non-Markovian Gaussian diffusive unravelings [L. Diósi and L. Ferialdi, Phys. Rev. Lett. 113, 200403 (2014)], here valid for arbitrary non-Hermitian system operators and noise correlations. The conditions under which the generalized stochastic Schrödinger equation has the same symmetry properties (invariance under unitary changes of operator base) than a microscopic system-bath Hamiltonian dynamics are determined. While the standard quantum diffusion model (standard noise correlations) always share the same invariance symmetry, the generalized stochastic dynamics can be mapped with an arbitrary bosonic environment only if some specific correlation constraints are fulfilled. These features are analyzed for different non-Markovian unravelings equivalent in average. Results based on quantum measurement theory that lead to specific cases of the generalized dynamics [J. Gambetta and H. M. Wiseman, Phys. Rev. A 66, 012108 (2002)] are studied from the perspective of the present analysis.

PACS numbers: 03.65.Yz, 03.65.Ta, 42.50.Lc, 05.40.Ca
\end{abstract}

\section{INTRODUCTION}

The theory of open quantum systems is well established when a Markovian approximation applies [1]. In this situation the density matrix evolution is defined by a Kossakovski-Lindblad equation. In addition, the system dynamics can be read in terms of an ensemble of stochastic trajectories developing in the system Hilbert space [1 9]. Different stochastic Schrödinger equations characterize the ensemble dynamics (unravelings). The diffusive case [2] corresponds to multiplicative Gaussian white noises. It allows to describe quantum systems subjected to a continuous measurement process [3 5] as well as to formulate dynamical wave vector collapse models [9].

In the last years, with the goal of establishing a nonMarkovian extension of the standard Markovian open quantum system theory, different research lines were opened. In particular, the stochastic ensemble representation of a quantum system coupled to a bosonic bath [10, 11] triggered the study of stochastic Schrödinger equations driven by multiplicative non-white Gaussian noises. As demonstrated in the seminal contributions of Strunz and Diósi [10], the emerging time-evolutionequation involves a functional derivative of the wave vector. Due to this feature, the formulation of different derivations and perturbation schemes, which lead to evolutions without involving an undetermined functional derivative, is a topic that is of interest up to present time [12 18]. On the other hand, exact closed expressions for the functional derivative were found in different physical situations [19 24].

The non-Markovian quantum diffusion model [10, 11] provided an alternative context for discussing nonMarkovian continuous measurement theory [25-27], nonMarkovian extensions of spontaneous wave function col- lapse models 28, 29], and operator correlations beyond the quantum regression theorem [30]. The formalism was also used to characterize specific physical systems such as for example quantum Brownian motion [31], complex optical arranges [32, 33], charge transport in organic crystals [34] and many body systems [35].

A relevant advance in the field was introduced recently by Diósi and Ferialdi [36]. On the basis of a path integral approach, they defined a generalized class of nonMarkovian Gaussian stochastic Schrödinger equations. Similarly to the Markovian case, the generalized unraveling is parametrized by a set of complex noise correlations that do not affect the density matrix evolution 7, 8]. For Markovian unravelings the degree of freedom introduced by the extra correlations can be set from symmetries constraints such as the invariance of the unraveling under linear system operator transformations under which the corresponding master equation is invariant [6 [8]. The main goal of this paper is to develop similar symmetry analysis for the generalized non-Markovian unraveling [36] and to find which constraints on the noise correlations arise.

We show that both the generalized non-Markovian Schrödinger equation and its associated density matrix evolution always share the same symmetry property, that is, they are invariant under arbitrary unitary changes of the system operator base. Therefore, in contrast to the Markovian case, we focus on which constraints on the noise correlations may arise when mapping the unravelling invariance with that of a microscopic system-bath Hamiltonian description. Different solutions to this problem are found, which are based on different kind of mapping between the noise and bath operator correlations. The emerging conditions under which the mapping is consistent are studied for a single dissipative channel dynamics. Furthermore, the stretched relation of the generalized non-Markovian unraveling with previous results 
derived from quantum-measurement theory [25] are analyzed from this perspective.

We base the present analysis on a generalized nonMarkovian Gaussian Schrödinger equation that, in contrast to Ref. [36], become written in an arbitrary nonHermitian base of system coupling operators. Instead of a path integral formalism, here the wave vector evolution [Eq. (12)] is derived by postulating a stochastic density matrix dynamics driven by multiplicative nonwhite Gaussian noises, where an undetermined contribution is obtained from a condition of average trace conservation [14].

The paper is organized as follows. In Sec. II, based on the stochastic approach outlined previously, we obtain the general non-Markovian Gaussian diffusive unraveling. The main results are obtained in Sec. III, where its symmetry properties are analyzed. In Sec. IV we exemplify the main results by studying the case of a single Hermitian coupling channel. Previous results obtained from quantum measurement theory are also studied and derived by using the present approach. The Conclusions are given in Sec. V. In the Appendix we define the main properties of the noise correlations as well as a generalization of Novikov theorem [37] valid for arbitrary multiplicative complex Gaussian noises.

\section{GENERAL STOCHASTIC GAUSSIAN DYNAMICS}

Both in the Markovian and non-Markovian regimes, stochastic Schrödinger equations define an ensemble of system states, which can be written in terms of a stochastic density matrix $\rho_{s t}(t)$. Its average over the ensemble of realizations, denoted as $\langle\cdots\rangle$, gives the system state, $\rho(t) \equiv\left\langle\rho_{s t}(t)\right\rangle$. In the present approach, we start by postulating the time evolution of $\rho_{s t}(t)$,

$$
\begin{aligned}
\frac{d}{d t} \rho_{s t}(t)= & -i\left[H_{S}, \rho_{s t}(t)\right]-\mathcal{U}\left[\rho_{s t}\right] \\
& -i \lambda\left(\mathcal{F}(t) \rho_{s t}(t)-\rho_{s t}(t) \mathcal{F}^{\dagger}(t)\right) .
\end{aligned}
$$

Here, $H_{S}$ is the system Hamiltonian. $\lambda$ is coupling parameter. The fluctuation operator reads

$$
\mathcal{F}(t) \equiv \sum_{\alpha} z_{\alpha}(t) L_{\alpha}
$$

where $\left\{L_{\alpha}\right\}$ is the set of coupling system operators. The set of (multiplicative) complex noises $\left\{z_{\alpha}(t)\right\}$ are Gaussian with null mean value $\left\langle z_{\alpha}(t)\right\rangle=0$, and correlations

$$
\chi_{\alpha \beta}(t, s) \equiv\left\langle z_{\alpha}^{*}(t) z_{\beta}(s)\right\rangle,
$$

and also

$$
\eta_{\alpha \beta}(t, s) \equiv\left\langle z_{\alpha}(t) z_{\beta}(s)\right\rangle .
$$

Notice that in contrast with previous analysis [14] and similarly to Ref. [36], here we are taking into account the correlations $\eta_{\alpha \beta}(t, s)$. These objects allow to consider arbitrary correlated noises (see Appendix). While the following derivation is similar to that when $\eta_{\alpha \beta}(t, s) \rightarrow 0$ 14], here we show it in order to enlighten the main assumptions over which it relies.

The unknown functional $\mathcal{U}\left[\rho_{s t}\right]$ is determined by average trace condition

$$
\frac{d}{d t} \operatorname{Tr}\left\langle\rho_{s t}(t)\right\rangle=0
$$

and the separability condition (pure state unraveling)

$$
\rho_{s t}(t)=|\psi(t)\rangle\langle\psi(t)| .
$$

This last requirement allow us to defining a stochastic Schrödinger equation for the system state $|\psi(t)\rangle$. In addition, this requisite implies that the stochastic map $\rho_{s t}(0) \rightarrow \rho_{s t}(t)$ is a completely positive one [1]. Therefore, Eq. (6) is a sufficient condition that guarantees the completely positive property of the density matrix evolution $\rho(0) \rightarrow \rho(t)$.

By averaging Eq. (10) and imposing condition (5) we get

$$
\operatorname{Tr}\left\langle\mathcal{U}\left[\rho_{s t}\right]\right\rangle=-i \lambda \operatorname{Tr}\left(\left\langle\mathcal{F}(t) \rho_{s t}(t)\right\rangle-\left\langle\rho_{s t}(t) \mathcal{F}^{\dagger}(t)\right\rangle\right) .
$$

Novikov theorem [37] gives an exact (functional) expression for the average of a product between a Gaussian noise and an arbitrary functional of it. In the Appendix we provide its generalization for arbitrary complex noises. Hence, from Eqs. (2) and (A.3) the previous contributions can be written as

$$
\begin{aligned}
\left\langle\mathcal{F}(t) \rho_{s t}(t)\right\rangle= & \int_{0}^{t} d s \chi_{\alpha \beta}^{*}(t, s) L_{\alpha}\left\langle\frac{\delta \rho_{s t}(t)}{\delta z_{\beta}^{*}(s)}\right\rangle \\
& +\int_{0}^{t} d s \eta_{\alpha \beta}(t, s) L_{\alpha}\left\langle\frac{\delta \rho_{s t}(t)}{\delta z_{\beta}(s)}\right\rangle,
\end{aligned}
$$

and similarly

$$
\begin{aligned}
\left\langle\rho_{s t}(t) \mathcal{F}^{\dagger}(t)\right\rangle= & \int_{0}^{t} d s \chi_{\alpha \beta}(t, s)\left\langle\frac{\delta \rho_{s t}(t)}{\delta z_{\beta}(s)}\right\rangle L_{\alpha}^{\dagger} \\
& +\int_{0}^{t} d s \eta_{\alpha \beta}^{*}(t, s)\left\langle\frac{\delta \rho_{s t}(t)}{\delta z_{\beta}^{*}(s)}\right\rangle L_{\alpha}^{\dagger} .
\end{aligned}
$$

The convention of sum over repeated subindices applies whenever the summatory symbol is not written. Given the commutativity of the trace operation, the indetermination given by Eq. (7) can be surpassed by demanding the separability condition (6), which due the analytical property of the wave vector $|\psi(t)\rangle$ implies

$$
\frac{\delta \rho_{s t}(t)}{\delta z_{\beta}(s)}=\frac{\delta|\psi(t)\rangle}{\delta z_{\beta}(s)}\left\langle\psi(t)\left|, \quad \frac{\delta \rho_{s t}(t)}{\delta z_{\beta}^{*}(s)}=\right| \psi(t)\right\rangle \frac{\delta\langle\psi(t)|}{\delta z_{\beta}^{*}(s)} .
$$

Therefore, the contribution $\mathcal{U}\left[\rho_{s t}\right]$ is determined in an unique way,

$\mathcal{U}\left[\rho_{s t}\right]=i \lambda \int_{0}^{t} d s\left(L_{\alpha}^{\dagger} \chi_{\alpha \beta}(t, s)-L_{\alpha} \eta_{\alpha \beta}(t, s)\right) \frac{\delta \rho_{s t}(t)}{\delta z_{\beta}(s)}+$ H.c., 
which in turn lead to the general non-Markovian Gaussian stochastic Schrödinger equation

$$
\begin{aligned}
\frac{d}{d t}|\psi(t)\rangle= & -i H_{S}|\psi(t)\rangle-i \lambda \mathcal{F}(t)|\psi(t)\rangle \\
& -i \lambda \int_{0}^{t} d s\left(L_{\alpha}^{\dagger} \chi_{\alpha \beta}(t, s)-L_{\alpha} \eta_{\alpha \beta}(t, s)\right) \frac{\delta|\psi(t)\rangle}{\delta z_{\beta}(s)} .
\end{aligned}
$$

This is the main result of this section. We notice that under the replacement $L_{\alpha}^{\dagger} \rightarrow L_{\alpha}$, this equation recovers the stochastic dynamics of Ref. 36]. As demonstrated in the next section, this replacement is equivalent to a unitary change of the base of system operators. Hence, Eq. (12) and the evolution obtained in [36] are equivalent dynamics expressed in different operator bases. On the other hand, when $\eta_{\alpha \beta}(t, s) \rightarrow 0$ it follows the standard non-Markovian quantum diffusion model [10, 14]. In the next section, we also show that the underlying symmetries of the generalized time evolution can be inferred and enlighten from Eq. (12) (Sec. III). In addition, this equivalent evolution allow us to recover in a simple way previous generalized stochastic dynamics obtained from quantum measurement theory [25] (Sec. IV).

\section{Functional structure and density matrix evolution}

As in the standard case [10, 14], we notice that Eq. (12) depends on the functional derivative of the wave vector. Most of the achievements performed in the last years [11 18] can be applied to the generalized unraveling. In particular, by following the calculation steps performed in Ref. [14] it follows

$$
\frac{\delta|\psi(t)\rangle}{\delta z_{\beta}(s)}=-i \lambda G_{s t}(t, s) L_{\beta}|\psi(s)\rangle,
$$

where the (functional) propagator is $G_{s t}(t, s)=$ $\left\lceil\exp -i \int_{s}^{t} d \tau \mathcal{T}_{s t}(\tau)\right\rceil$. Here, $\lceil\cdots\rceil$ denotes a time ordering operation while the functional generator $\mathcal{T}_{s t}(t)$ defines the time evolution (12), that is, $(d / d t)|\psi(t)\rangle=$ $\mathcal{T}_{s t}(t)|\psi(t)\rangle$. As demonstrated in Ref. [14] these expressions allow us to perform consistent approximations (in the interaction parameter or in the noise correlation times) of the stochastic wave vector in both a time convoluted and time convolutionless schemes.

By introducing Eq. (11) in the stochastic evolution (11) and after performing the average over realizations [see Eqs. (8) and (9)], we obtain the density matrix evolution

$$
\begin{aligned}
\frac{d}{d t} \rho(t)= & -i\left[H_{S}, \rho(t)\right]-i \lambda \int_{0}^{t} d s\left\{\chi_{\alpha \beta}(t, s)\right. \\
& \left.\times\left[L_{\alpha}^{\dagger},\left\langle\frac{\delta \rho_{s t}(t)}{\delta z_{\beta}(s)}\right\rangle\right]-\text { H.c. }\right\}
\end{aligned}
$$

where the functional derivative is given by

$$
\frac{\delta \rho_{s t}(t)}{\delta z_{\beta}(s)}=-i \lambda G_{s t}(t, s) L_{\beta} \rho_{s t}(s) G_{s t}^{\dagger}(t, s),
$$

result that follows from Eq. (13).

We notice that Eq. (14) does not depend explicitly on the correlations $\eta_{\alpha \beta}(t, s)$. By performing a recursive expansion in the interaction strength parameter $\lambda$ [14], it is also possible to demonstrate that the density matrix evolution, order by order in $\lambda$, does not depend of $\eta_{\alpha \beta}(t, s)$. Therefore, consistently with Ref. [36], we conclude that these correlations only modify the wave vector evolution. Their physical role is investigated through the following symmetry analysis.

\section{SYMMETRIES}

Here, we analyze the symmetries of the generalized stochastic Schrödinger evolution defined by Eq. (12).

\section{A. Hermitian fluctuations}

When the fluctuation operator (2) is Hermitian

$$
\mathcal{F}^{\dagger}(t)=\mathcal{F}(t)
$$

it follows the equality

$$
\sum_{\alpha} L_{\alpha}^{\dagger} \chi_{\alpha \beta}(t, s)=\sum_{\alpha} L_{\alpha} \eta_{\alpha \beta}(t, s) .
$$

This relation follows by multiplying Eq. (16) by $z_{\beta}(s)$ and using the definition of the noise correlations, Eqs. (3) and (4). We notice that the weight of the functional contribution in Eq. (12) is precisely the difference between the two terms in the equality (17). Hence, it consistently vanishes for Hermitian fluctuations.

\section{B. Invariance under unitary changes of system operator base}

In the derivation of Sec. II the fluctuation operator $\mathcal{F}(t)$ was written in the base defined by the set of operators $\left\{L_{\alpha}\right\}$. Here, we explore which structure assume the evolution (12) when introducing a new base of operators $\left\{A_{\mu}\right\}$ related to the previous one by a unitary transformation. Hence, we write

$$
A_{\mu}=\sum_{\alpha} L_{\alpha} U_{\alpha \mu}, \quad L_{\alpha}=\sum_{\mu} A_{\mu} U_{\mu \alpha}^{\dagger}
$$

where the unitary operator $U$ satisfies $\sum_{\mu} U_{\alpha \mu} U_{\mu \beta}^{\dagger}=\delta_{\alpha \beta}$ and $\sum_{\alpha} U_{\mu \alpha}^{\dagger} U_{\alpha \nu}=\delta_{\mu \nu}$. This change of base allows to define a new set of noises $\left\{r_{\mu}(t)\right\}$ related to the original one as

$$
r_{\mu}(t)=\sum_{\alpha} U_{\mu \alpha}^{\dagger} z_{\alpha}(t), \quad z_{\alpha}(t)=\sum_{\mu} U_{\alpha \mu} r_{\mu}(t),
$$


such that the fluctuation operator can be rewritten as

$$
\mathcal{F}(t)=\sum_{\alpha} z_{\alpha}(t) L_{\alpha}=\sum_{\mu} r_{\mu}(t) A_{\mu} .
$$

The variational derivative in Eq. (12) can be written as

$$
\frac{\delta(\cdot)}{\delta z_{\beta}(s)}=\sum_{\nu} \int_{0}^{\infty} d \tau \frac{\delta(\cdot)}{\delta r_{\nu}(\tau)} \frac{\delta r_{\nu}(\tau)}{\delta z_{\beta}(s)}=\sum_{\nu} U_{\nu \beta}^{\dagger} \frac{\delta(\cdot)}{\delta r_{\nu}(s)}
$$

where we have used that $\delta r_{\nu}(\tau) / \delta z_{\beta}(s)=\delta(\tau-s) U_{\nu \beta}^{\dagger}$ [Eq. (19)]. Hence, from the previous expressions, in the new base the general stochastic Schrödinger equation becomes

$$
\begin{aligned}
\frac{d}{d t}|\psi(t)\rangle= & -i H_{S}|\psi(t)\rangle-i \lambda \mathcal{F}(t)|\psi(t)\rangle \\
& -i \lambda \int_{0}^{t} d s\left(A_{\mu}^{\dagger} \chi_{\mu \nu}(t, s)-A_{\mu} \eta_{\mu \nu}(t, s)\right) \frac{\delta|\psi(t)\rangle}{\delta r_{\nu}(s)}
\end{aligned}
$$

where we have defined the correlations

$$
\chi_{\mu \nu}(t, s) \equiv \sum_{\alpha \beta} U_{\alpha \mu} \chi_{\alpha \beta}(t, s) U_{\nu \beta}^{\dagger}=\left\langle r_{\mu}^{*}(t) r_{\nu}(s)\right\rangle,
$$

and also

$$
\eta_{\mu \nu}(t, s) \equiv \sum_{\alpha \beta} U_{\mu \alpha}^{\dagger} \eta_{\alpha \beta}(t, s) U_{\nu \beta}^{\dagger}=\left\langle r_{\mu}(t) r_{\nu}(s)\right\rangle .
$$

Equation (22) and the previous two expressions show that the general evolution (12) is invariant under the unitary changes defined by Eqs. (18) and (19). One can always choose an Hermitian base of operators $\left\{A_{\mu}\right\}=$ $\left\{A_{\mu}^{\dagger}\right\}$, which explicitly demonstrate that Eq. (12) and Eq. (27) in Ref. [36] are related by a unitary change of operator bases. On the other hand, from Eq. (21) it is possible to demonstrate that the density matrix evolution (14) is also invariant under the transformation (18) and (19), that is, it can be rewritten in terms of the operators $A_{\mu}$, the derivative $\delta \rho_{s t}(t) / \delta r_{\nu}(s)$, and the correlations $\chi_{\mu \nu}(t, s)[\mathrm{Eq} \cdot(23)]$.

Given that $\chi_{\mu \nu}(t, s)$ and $\eta_{\mu \nu}(t, s)$ only depend on the correlations $\chi_{\alpha \beta}(t, s)$ and $\eta_{\alpha \beta}(t, s)$ respectively, the independence of $\rho(t)$ with respect to $\eta_{\alpha \beta}(t, s)$ [Eq. (14)] also implies its independence with respect to $\eta_{\mu \nu}(t, s)$ in the new base. Therefore, this property is valid in any operator basis, conclusion consistent with the results of Ref. [36].

\section{White noises}

For white correlated noises

$$
\chi_{\alpha \beta}(t, s)=\delta(t-s) \delta_{\alpha \beta}, \quad \eta_{\alpha \beta}(t, s)=\delta(t-s) c_{\alpha \beta},
$$

where $c_{\alpha \beta}$ are complex coefficients, by using Eqs. (14) and (15), the density matrix evolution becomes a Lindblad equation

$$
\frac{d \rho(t)}{d t}=-i\left[H_{S}, \rho(t)\right]+\lambda^{2}\left(\left[L_{\alpha}, \rho(t) L_{\alpha}^{\dagger}\right]+\left[L_{\alpha} \rho(t), L_{\alpha}^{\dagger}\right]\right)
$$

Under the unitary transformation (18), this equation remains invariant, that is, the only change corresponds to the replacements $L_{\alpha} \rightarrow A_{\mu}$. On the other hand, in addition to this change, Eq. (22) results defined by the correlations [see Eqs. (23) and (24)]

$$
\begin{aligned}
& \chi_{\mu \nu}(t, s)=\delta(t-s) \delta_{\mu \nu}, \\
& \eta_{\mu \nu}(t, s)=\delta(t-s) \sum_{\alpha \beta} U_{\mu \alpha}^{\dagger} c_{\alpha \beta} U_{\nu \beta}^{\dagger} .
\end{aligned}
$$

While the master equation is invariant (does not depend explicitly) under the unitary transformation $U$, the stochastic evolution depends on it through the correlation $\eta_{\mu \nu}(t, s)$. Therefore, if one demand that both the master equation and the stochastic Schrödinger equation must have the same dependence on $U$ (symmetry) it follows that $c_{\alpha \beta} \rightarrow 0$. Hence, $\eta_{\alpha \beta}(t, s) \rightarrow 0$. Based on a different approach, this result was developed in Ref. [7]. Now, we ask if this kind of arguments are also applicable for the generalized non-Markovian Schrödinger equation.

\section{Mapping with microscopic Hamiltonian symmetries}

The previous analysis (valid for white noises) does not apply in the present framework. In fact, a redefinition of the noises allowed us to conclude that the generalized Schrödinger dynamics (12) and the master equation (14) share the same invariance symmetry property under unitary changes of the operator bases. Thus, any constraint applicable to the noise correlations has to be based on a more fundamental requirement. With this motivation, we ask if the stochastic Schrödinger equation has the same invariance symmetry property than a microscopic bosonic dynamics able to induce the same system dynamics.

The total microscopic system-reservoir Hamiltonian $H_{T}$ reads

$$
H_{T}=H_{S}+H_{B}+\lambda \sum_{\alpha} L_{\alpha} \otimes Z_{\alpha}
$$

where as before $H_{S}$ is the system Hamiltonian, $H_{B}$ is the bath Hamiltonian and the remaining contribution gives their interaction. Similarly, we introduce a unitary change of the system operator base [Eq. (18)]

$$
A_{\mu}=\sum_{\alpha} L_{\alpha} U_{\alpha \mu}, \quad L_{\alpha}=\sum_{\mu} A_{\mu} U_{\mu \alpha}^{\dagger},
$$

and a new set of bath operators

$$
R_{\mu}=\sum_{\alpha} U_{\mu \alpha}^{\dagger} Z_{\alpha}, \quad Z_{\alpha}=\sum_{\mu} U_{\alpha \mu} R_{\mu},
$$

in such a way that the interaction contribution $H_{I}$ can be written as

$$
H_{I}=\sum_{\alpha} L_{\alpha} \otimes Z_{\alpha}=\sum_{\mu} A_{\mu} \otimes R_{\mu} .
$$


When tracing out the bath dynamics, $\rho(t)=$ $\operatorname{Tr}_{B}\left[\exp \left(-i t H_{T}\right) \rho(0) \otimes \rho_{B} \exp \left(+i t H_{T}\right)\right]$, where $\operatorname{Tr}_{B}[\cdots]$ is a trace operation in the bath Hilbert space and $\rho_{B}$ is the environment stationary state, given that $\operatorname{Tr}_{B}\left[\rho_{B} Z_{\alpha}(t)\right]=$ $\operatorname{Tr}_{B}\left[\rho_{B} \exp \left(+i t H_{B}\right) Z_{\alpha} \exp \left(-i t H_{B}\right)\right]=0$, the relevant statistical objects are the bath operator correlations (in an interaction picture with respect to $H_{B}$ ) [1, 4]. Therefore, we search under which conditions both noise correlations $\chi_{\alpha \beta}(t, s)$ and $\eta_{\alpha \beta}(t, s)$ can be related or mapped with the quantum bath correlations.

Some conditions are imposed over the bath-noise correlation mapping.

(i) The map has to be invariant under unitary changes of the system operator base.

(ii) The density matrix evolution obtained from the stochastic and microscopic Hamiltonian approaches must be the same.

(iii) In addition, the mapping must be consistent, that is, the resulting noise correlation matrix has to be positive defined [see Eq. (A.4) in the Appendix].

Under the previous conditions, different mapping with different physical motivations can be proposed. A diagonal map arises from a direct mapping between the stochastic and microscopic dynamics, which lead to the condition $\eta_{\alpha \beta}(t, s) \rightarrow 0$. A non-diagonal map is motivated from quantum measurement theory, which allows us to raise the previous condition, $\eta_{\alpha \beta}(t, s) \neq 0$, if some constraints are fulfilled. The diagonal mapping can be read as a particular case of the non-diagonal one. Nevertheless, for clarity, below each case is presented in a separate way.

\section{Diagonal correlation mapping}

The stochastic and microscopic density matrix dynamics can be put in one-to-one correspondence [see Eqs. (20) and [30] ] under the following correlation associations

$$
\begin{aligned}
\chi_{\alpha \beta}(t, s) & \leftrightarrow \operatorname{Tr}_{B}\left[\rho_{B} Z_{\alpha}^{\dagger}(t) Z_{\beta}(s)\right], \\
\eta_{\alpha \beta}(t, s) & \leftrightarrow \operatorname{Tr}_{B}\left[\rho_{B} Z_{\alpha}(t) Z_{\beta}(s)\right] .
\end{aligned}
$$

This correlation map is invariant under unitary changes of system operator base [condition (i)]. In fact, from Eqs. (19) and (29), in the new base Eq. (31) becomes

$$
\begin{aligned}
\chi_{\mu \nu}(t, s) & \leftrightarrow \operatorname{Tr}_{B}\left[\rho_{B} R_{\mu}^{\dagger}(t) R_{\nu}(s)\right], \\
\eta_{\mu \nu}(t, s) & \leftrightarrow \operatorname{Tr}_{B}\left[\rho_{B} R_{\mu}(t) R_{\nu}(s)\right],
\end{aligned}
$$

which corresponds to the same mapping in the new noise and bath operator bases.

Given that the average density matrix does not depend on the correlations $\eta_{\alpha \beta}(t, s)$, the equality $\chi_{\alpha \beta}(t, s)=$ $\operatorname{Tr}_{B}\left[\rho_{B} Z_{\alpha}^{\dagger}(t) Z_{\beta}(s)\right]$ guarantees condition (ii), that is, the stochastic and microscopic approach lead to the same density matrix evolution (see Ref. 14]). Notice that due to the Gaussian and bosonic properties of the fluctuation operator and reservoir respectively, it is not necessary to define the map for higher correlations.
The map (31) should allows us to associate a microscopic origin to both noise correlations $\chi_{\alpha \beta}(t, s)$ and $\eta_{\alpha \beta}(t, s)$. Nevertheless, by taking the relations (31) as valid equalities, by using that $H_{I}^{\dagger}(t)=H_{I}(t)$, from Eq. (17) one immediately deduce (in an interaction representation) that the fluctuation operator $\mathcal{F}(t)$ should be Hermitian, implying the cancellation of the variational contribution in the stochastic Schrödinger evolution (12). Hermitian fluctuations can only be mapped with a quantum reservoir at infinite temperature [14, 38]. Given an environment at finite temperature, this contradiction can only be surpassed by imposing the correlation map

$$
\chi_{\alpha \beta}(t, s)=\operatorname{Tr}_{B}\left[\rho_{B} Z_{\alpha}^{\dagger}(t) Z_{\beta}(s)\right],
$$

and demanding

$$
\eta_{\alpha \beta}(t, s)=0
$$

In the Appendix we demonstrate that, without imposing any condition on the bath properties, these correlations satisfy condition (iii). Therefore, Eq. (33) and (34) define a consistent correlation map which in turn recovers the standard non-Markovian quantum diffusion model 10, 11] (Eq. (12) with $\left.\eta_{\alpha \beta}(t, s) \rightarrow 0\right)$. This is one of the main results of this section. Consistently, Eq. (34) also implies $\eta_{\mu \nu}(t, s)=0$.

The previous correlation map [Eqs. (33) and (34)] guaranties that the stochastic and microscopic evolution (at any temperature) have the same invariance symmetry property under arbitrary unitary changes of the system operator base. Notice that the alternative mapping $\eta_{\alpha \beta}(t, s)=\operatorname{Tr}_{B}\left[\rho_{B} Z_{\alpha}(t) Z_{\beta}(s)\right]$ with $\chi_{\alpha \beta}(t, s)=0$ is not consistent with condition (iii) [see Eq. [A.4] ]. In fact, for arbitrary complex noises it follows $\left.\chi_{\alpha \beta}(t, s)\right|_{t=s, \alpha=\beta}>0$.

\section{Non-diagonal (measurement-like) correlation mapping}

In diverse quantum optical arranges, the Markovian dynamics of a system is inferred from a measurement process performed on the environment. Different measurement schemes are defined by the quadratures of the bath, which in turn define different stochastic unravelings [3, 4] with the same average dynamics. In the present context, a similar degree of freedom can be introduced by defining a set of quadrature-like [3, 4] bath operators $\left\{\mathcal{Z}_{\alpha}(t)\right\}$, which read

$$
\mathcal{Z}_{\alpha}(t) \equiv \sum_{\alpha^{\prime}} M_{\alpha \alpha^{\prime}} Z_{\alpha^{\prime}}(t)
$$

Here $M_{\alpha \alpha^{\prime}}$ is an arbitrary complex matrix. When $M_{\alpha \alpha^{\prime}}=\delta_{\alpha \alpha^{\prime}}$ the interaction bath operators [see Eq. (27)] are recovered. Therefore, consistently with the previous diagonal case, maintaining the base of system operators $\left\{L_{\alpha}\right\}$, instead of Eq. (31), we introduce the generalized 
correlation mapping

$$
\begin{aligned}
\chi_{\alpha \beta}(t, s) & =\operatorname{Tr}_{B}\left[\rho_{B} \mathcal{Z}_{\alpha}^{\dagger}(t) \mathcal{Z}_{\beta}(s)\right] \\
\eta_{\alpha \beta}(t, s) & =\operatorname{Tr}_{B}\left[\rho_{B} \mathcal{Z}_{\alpha}(t) \mathcal{Z}_{\beta}(s)\right] .
\end{aligned}
$$

The non-diagonal associations (36) are also invariant under changes of the system operator base [condition (i)]. In fact, from the Schrödinger evolution (22), Eq. (28) and (29), it follows Eq. (32) with the replacement $R_{\mu}(t) \rightarrow$ $\mathcal{R}_{\mu}(t)$, where

$$
\mathcal{R}_{\mu}(t)=M_{\mu \mu^{\prime}} R_{\mu^{\prime}}(t)=\left(U_{\mu \alpha}^{\dagger} M_{\alpha \alpha^{\prime}} U_{\alpha^{\prime} \mu^{\prime}}\right) R_{\mu^{\prime}}(t) .
$$

Hence, invariance is guaranteed by a unitary transformation of the matrix $M_{\alpha \alpha^{\prime}}$.

Condition (ii) implies that the density matrix evolution has to remains the same than in the diagonal case, which in fact corresponds to the dynamics derived from the microscopic Hamiltonian dynamics. Given that in Eqs. (31) and (36) the base of system operators is the same, and given that the density matrix evolution (14) does not depend on the correlations $\eta_{\alpha \beta}(t)$ [36], it follows the condition

$$
\operatorname{Tr}_{B}\left[\rho_{B} Z_{\alpha}^{\dagger}(t) Z_{\beta}(s)\right]=\operatorname{Tr}_{B}\left[\rho_{B} \mathcal{Z}_{\alpha}^{\dagger}(t) \mathcal{Z}_{\beta}(s)\right] .
$$

Therefore, the bath operators $\left\{Z_{\alpha}(t)\right\}$ and the quadrature-like bath operators $\left\{\mathcal{Z}_{\alpha}(t)\right\}$ must have the same correlations. From Eq. (35) this condition explicitly reads

$$
\operatorname{Tr}_{B}\left[\rho_{B} Z_{\alpha}^{\dagger}(t) Z_{\beta}(s)\right]=M_{\alpha^{\prime} \alpha}^{\dagger} M_{\beta \beta^{\prime}} \operatorname{Tr}_{B}\left[\rho_{B} Z_{\alpha^{\prime}}^{\dagger}(t) Z_{\beta^{\prime}}(s)\right] .
$$

Even when the previous constraint is fulfilled, it is not possible to guaranty that condition (iii) is satisfied. That is, it is not possible to know in general if the quantum correlations defined by $\left\{\mathcal{Z}_{\alpha}(t)\right\}[$ Eq. (36) $]$ satisfy or not the positivity condition given by Eq. (A.4).

In general, it may be difficult to satisfy the previous requirements without imposing some condition on the matrix $M_{\alpha \beta}$ or alternatively on the properties of the bath. Notice that in the white noise approximation $\operatorname{Tr}_{B}\left[\rho_{B} Z_{\alpha}^{\dagger}(t) Z_{\beta}(s)\right]=\delta(t-s) \delta_{\alpha \beta}[\mathrm{Eq}$. (25)], Eq. (38) is automatically satisfied for unitary matrixes, $M_{\alpha^{\prime} \alpha}^{\dagger} M_{\beta \alpha^{\prime}}=\delta_{\alpha \beta}$ (Stratonovich calculus).

Assuming the consistence of the map (36) with conditions (ii) and (iii), in contrast to the diagonal case, here the Hermiticity of the interaction Hamiltonian $H_{I}(t)$ and Eq. (17) do not lead in general to any contradiction. Therefore, given that Eqs. (36) are consistent with the correlations of a set of complex noises, and given that Eq. (38) is satisfied, the non diagonal mapping [Eq. (35)] allow us to define the correlations of the generalized Eq. (12) from the microscopic underlying evolution. This is the second main result of this section. In fact, we obtained conditions under which the different unravelings related to Eq. (12) can be associated to the microscopic Hamiltonian dynamics. At this stage, notice that the diagonal case can be read as a particular case of the present one.

\section{EXAMPLES}

In the next examples we analyze the consequences of the previous constraints when applied to different system-bath interaction structures. The relation of the generalized stochastic unraveling with previous results based on quantum-measurement theory [25] is also revisited.

\section{A. Hermitian single channel}

The general evolution (12) takes a simpler form when one take into account only one single channel, $\mathcal{F}(t)=$ $z(t) L$, defined by an Hermitian operator, $L^{\dagger}=L$. Therefore, it follows

$$
\begin{aligned}
\frac{d}{d t}|\psi(t)\rangle= & -i H_{S}|\psi(t)\rangle-i \lambda z(t) L|\psi(t)\rangle \\
& -i \lambda L \int_{0}^{t} d s(\chi(t, s)-\eta(t, s)) \frac{\delta|\psi(t)\rangle}{\delta z(s)} .
\end{aligned}
$$

From a phenomenological point of view, one can propose different noise correlations $\chi(t, s)$ and $\eta(t, s)$, as for example exponential ones. In this case, it is possible to explicitly show [see Appendix, Eq. A.9 ] that the extra correlation $\eta(t, s)$ allows to smoothly change the stochastic unraveling between the standard non-Markovian quantum diffusion model $[\eta(t, s)=0]$ and a pure stochastic Hamiltonian $[\eta(t, s)=\chi(t, s)]$. On the other hand, the consistency of this evolution with the invariance symmetry of a microscopic Hamiltonian description is analyzed below.

Eq. (39) should be associated with an interaction Hamiltonian of the form $H_{I}=L \otimes Z$, where the bath operator is Hermitian, $Z=Z^{\dagger}$, with (complex) correlation $\operatorname{Tr}_{B}\left[\rho_{B} Z(t) Z(s)\right]$. If one try to impose the associations (31), it follows that $\chi(t, s)=\eta(t, s)$. This equality can only be satisfied by a real noise $z(t)$ with a real correlation [see Eq. A.4 ]. Consequently $\mathcal{F}^{\dagger}(t)=\mathcal{F}(t)$. Given that $\chi(t, s)=\chi^{*}(t, s)=\eta(t, s)=\eta^{*}(t, s)$, it also implies $\operatorname{Tr}_{B}\left[\rho_{B} Z(t) Z(s)\right]=\operatorname{Tr}_{B}\left[\rho_{B} Z(s) Z(t)\right]$. In general, this equality can only be satisfied when $\rho_{B}$ is proportional to the identity matrix, that is, a thermal state at infinite temperature. This contradiction, which also implies the cancellation of the functional contribution in Eq. (39), is raised up by taking $\chi(t, s)=\operatorname{Tr}_{B}\left[\rho_{B} Z(t) Z(s)\right]$ and $\eta(t, s)=0$, that is, consitently we recover the diagonal correlation mapping defined by Eqs. (33) and (34) respectively.

Given that the interaction Hamiltonian only involves one single Hermitian operator $Z$, here it is not possible to introduce a non-diagonal correlation mapping (36). Therefore, the correlation $\eta(t, s)$ in Eq. (39) cannot be related to the microscopic interaction if the same invariance symmetry property is demanded. 


\section{Dephasing dynamics}

Dephasing dynamics is one particular physical case of the previous situation. It emerges when the (Hermitian) coupling operator commutates with the system Hamiltonian, $\left[H_{S}, L\right]=0$. Hence, in Eq. (39), the variational derivative can be written as

$$
\frac{\delta|\psi(t)\rangle}{\delta z(s)}=-i \lambda L|\psi(t)\rangle
$$

result that follows straightforward from Eq. (13). On the other hand, from Eqs. (14) and (15) it follows the density matrix evolution

$$
\frac{d}{d t} \rho(t)=-i\left[H_{S}, \rho(t)\right]-\lambda^{2} \int_{0}^{t} d s \kappa(t, s)[L,[L, \rho(t)]]
$$

where $\kappa(t, s)=\left[\chi(t, s)+\chi^{*}(t, s)\right] / 2$. We notice that this time evolution also arises from a pure stochastic Hamiltonian (see Eq. (40) in Ref. [38]) defined with a real Gaussian noise with correlation $\kappa(t, s)$. Therefore, all the set of ensembles defined by Eq. (39) and (40) are equivalent in average to a stochastic Hamiltonian. This property is stretchy related to the dephasing property of the underlying fluctuations.

\section{B. Quantum optical-like microscopic interaction}

For quantum optical systems, where a Markovian approximation applies, the correlations $\eta_{\alpha \beta}(t, s)$ (delta correlated) define different ensemble dynamics associated to different measurement processes performed over the environmental degrees of freedom [8]. Based on quantum measurement theory, Gambetta and Wiseman demonstrated that a similar result is valid in the non-Markovian regime [25]. Below, we show that those results can be recovered as diagonal and non-diagonal correlation mappings associated to the same microscopic Hamiltonian. In fact, the degree of freedom associated to the different measurement processes here can be related to alternative definitions of the bath operators $\left\{\mathcal{Z}_{\alpha}(t)\right\}$ [Eq. (35)]

The system Hamiltonian is split as $H_{S}=H_{0}+H$. The bath Hamiltonian is taken as $H_{B}=\sum_{k} \omega_{k} a_{k}^{\dagger} a_{k}$, where $a_{k}^{\dagger}$ and $a_{k}$ are bosonic creation and annihilation operators respectively, $\omega_{k}$ the frequency of each mode. The system-bath interaction is $H_{I}=i\left(L \otimes b^{\dagger}-L^{\dagger} \otimes b\right)$, with $b=\sum_{k} g_{k} a_{k}$, where $g_{k}$ are coupling constants. The stationary bath density matrix $\rho_{B}$ is the vacuum state. In an interaction representation with respect to $H_{0}$ and $H_{B}$, it follows

$$
H_{I}(t)=(i L) Z^{\dagger}(t)+\left(-i L^{\dagger}\right) Z(t),
$$

where the bath operator is $Z(t)=\sum_{k} g_{k} a_{k} e^{-i \Omega_{k} t}$, with $\Omega_{k} \equiv \omega_{k}-\omega_{0}$. The frequency $\omega_{0}$ follows from the assumption $L(t)=e^{+i t H_{0}} L e^{-i t H_{0}}=L e^{-i \omega_{0} t}$.
Noticing that $\left\{Z_{\alpha}(t)\right\}=\left\{Z^{\dagger}(t), Z(t)\right\}$ characterize the interaction Hamiltonian in the base of system operators $\left\{L_{\alpha}\right\}=\left\{i L,-i L^{\dagger}\right\}$, demanding the positivity of the noise correlation matrix [see Eq. (A.4)], it is simple to check that the diagonal correlation mapping defined by Eqs. (33) and (34) can only be consistently satisfied by introducing one single complex noise $z_{c}(s), \mathcal{F}(t)=z_{c}(t)(i L)$, such that $\chi_{c}(t, s)=\left\langle z_{c}^{*}(t) z_{c}(s)\right\rangle=\operatorname{Tr}_{B}\left[\rho_{B} Z(t) Z^{\dagger}(s)\right]=$ $\sum_{k}\left|g_{k}\right|^{2} e^{-i \Omega_{k}(t-s)}$, and $\eta_{c}(t, s)=\left\langle z_{c}(t) z_{c}(s)\right\rangle=0$. Hence, Eq. (12) becomes $\left[H(t)=e^{+i t H_{0}} H e^{-i t H_{0}}\right]$

$$
\begin{aligned}
\frac{d}{d t}|\psi(t)\rangle= & -i H(t)|\psi(t)\rangle+z_{c}(t) L|\psi(t)\rangle \\
& -L^{\dagger} \int_{0}^{t} d s \chi_{c}(t-s) \frac{\delta|\psi(t)\rangle}{\delta z_{c}(s)}
\end{aligned}
$$

From quantum measurement theory, this evolution can be read as a coherent unravelling of the bath (see Eq. (3.22) in [25]) where $Z(t)$ is the noise operator. A heterodyne measurement process is recovered in the Markovian limit [25].

As an example of non-diagonal mapping, Eq. (35), we take the single operator $\mathcal{Z}(t)=Z(t)+Z^{\dagger}(t)$, and consistently with the diagonal case $\mathcal{F}(t)=z_{q}(t)(i L)$. It is simple to check that Eq. (38) is fulfilled. In fact, given that $\mathcal{Z}^{\dagger}(t)=\mathcal{Z}(t)$, Eq. (36) leads to the correlations $\chi_{q}(t, s)=\eta_{q}(t, s)=\operatorname{Tr}_{B}\left[\rho_{B} \mathcal{Z}(t) \mathcal{Z}(s)\right]=$ $\sum_{k}\left|g_{k}\right|^{2} e^{-i \Omega_{k}(t-s)}$ (the same correlation than in the coherent unraveling). Nevertheless, these correlations do not have associated a positive covariance matrix [Eq. (A.4)]. This contradiction can be raised up by taking the complex noise $z_{q}(t)$ as a real one, implying real correlations. Given that $L \neq L^{\dagger}$, here a real noise does not implies $\mathcal{F}^{\dagger}(t)=\mathcal{F}(t)$. The previous complex correlations become real if we demand extra properties to the bath. If $\Omega_{-k}=-\Omega_{k}$ and, given that $H_{I}^{\dagger}(t)=H_{I}(t)$, demanding $g_{-k}=g_{k}^{*}$, it follows $\chi_{q}(t, s)=\eta_{q}(t, s)=$ $2 \sum_{k>0}\left|g_{k}\right|^{2} \cos \left[\Omega_{k}(t-s)\right] \equiv \beta_{q}(t-s)$. Therefore, from Eq. (12) we arrive to the generalized Schrödinger equation

$$
\begin{aligned}
\frac{d}{d t}|\psi(t)\rangle= & -i H(t)|\psi(t)\rangle+z_{q}(t) L|\psi(t)\rangle \\
& -\left(L^{\dagger}+L\right) \int_{0}^{t} d s \beta_{q}(t-s) \frac{\delta|\psi(t)\rangle}{\delta z_{q}(s)} .
\end{aligned}
$$

This evolution can be read as a quadrature unravelling of the bath (see Eq. (4.30) in [25]) where $Z(t)+Z^{\dagger}(t)$ is the noise operator. A homodyne measurement process is recovered in the Markovian limit [25].

We derived Eqs. (43) and (44) on the basis of the diagonal and non-diagonal correlation mapping introduced previously. These results give a solid support to the present and previous analysis [25, 26], which rely on quantum measurement theory.

Based on the analysis of Refs. [25, 26], we also conclude that the ensemble of realizations associated to generalized Schrödinger dynamics such as Eq. (12) cannot be read as conditional states of a system subjected to 
a continuous measurement process over the environment degrees of freedom. In fact, as argued in Refs. [25, 26], in the non-Markovian regime the wave vector only represents the state the system would be at a single time. The property of linking solutions at different times to make a trajectory of a continuously monitored system is lost [27].

\section{SUMMARY AND CONCLUSIONS}

We based the present analysis on a stochastic approach. Postulating an underlying evolution with multiplicative noises, we derived the generalized nonMarkovian Gaussian stochastic Schrödinger equation (12) from the condition of average trace preservation. Complex Gaussian noises with arbitrary correlations $\chi_{\alpha \beta}(t, s)$ and $\eta_{\alpha \beta}(t, s)$, Eqs. (3) and (4) respectively, were considered.

We focused our study on the symmetries of the obtained evolution. The generalized Schrödinger equation, after a redefinition of the noises, is invariant under arbitrary unitary changes of the system operator base. This property allowed us to conclude that Eq. (12) and the stochastic dynamics derived in Ref. [36] are related by a unitary transformation. On the other hand, the associated master equation share the same symmetry property than the stochastic Schrödinger equation. Therefore, in contrast to previous analysis with Markovian dynamics, we asked under which conditions the invariance property can be mapped with that of a microscopic (bosonian) system-bath dynamics, which in turn lead us to consider a mapping between the noise correlations $\chi_{\alpha \beta}(t, s)$ and $\eta_{\alpha \beta}(t, s)$ with the bath operator correlations.

Two kind of maps were introduced. In the diagonal one, the quantum reservoir correlations are defined by the bath operators corresponding to the system-environment interaction Hamiltonian. In the non-diagonal map, a new set of bath operators is introduced (quadrature-like bath operators), being related to the previous ones by an arbitrary linear transformation.

For the diagonal correlation mapping [Eq. (31)], the invariance symmetry property of the microscopic dynamics is shared by the stochastic unravelling only when the correlation $\eta_{\alpha \beta}(t, s)$ vanishes, Eqs. (33) and (34), recovering in consequence the standard non-Markovian quantum diffusion model [10]. For the non-diagonal correlation map [Eq. (36)], the invariance symmetry is shared only if some constraints are fulfilled. In fact, the independence of the density matrix evolution with respect to $\eta_{\alpha \beta}(t, s)$ and the positivity of the noise correlation matrix lead to the necessary conditions Eqs. (38) and (A.4) respectively. These requirements establish the conditions under which the noise correlations that set the generalized unraveling, $\chi_{\alpha \beta}(t, s)$ and $\eta_{\alpha \beta}(t, s)$, can be defined from the properties of the microscopic system-bath interaction.

As example, we considered the case of a single noise channel defined by an Hermitian operator [Eq. (39)]. We concluded that only the standard version of the dynamics can be related with a microscopic description if the invariance property is demanded. On the other hand, previous dynamics derived from quantum measurement theory were recovered from diagonal and non-diagonal correlation mapping [Eqs. (43) and (44) respectively] applied to the same optical-like system-environment interaction [25]. These examples show that the consistence of the non-diagonal correlation map put severe constraints on the bath properties, which in general may or not be fulfilled.

The present analysis not only clarifies the recent advances in the formulation of Gaussian stochastic wave vector dynamics 36 but also define the constraints under which the generalized unraveling can be put in one-to-one correspondence with a microscopic unitary description. They also supports the stringent constraints on the interpretation of non-Markovian Schrödinger equations as conditional measurement states at different times [27].

\section{Acknowledgment}

This work was supported by Consejo Nacional de Investigaciones Científicas y Técnicas (CONICET), Argentina.

\section{Appendix: Arbitrary complex Gaussian noises}

The statistical properties of an arbitrary set of complex Gaussian noises $\left\{z_{\alpha}(t)\right\}$ can be defined through the characteristic functional

$$
G[f, g] \equiv\left\langle\exp \left\{i \int_{0}^{t} d \tau\left[f_{\alpha}(\tau) z_{\alpha}(\tau)+g_{\alpha}(\tau) z_{\alpha}^{*}(\tau)\right]\right\}\right\rangle
$$

where $f$ and $g$ denotes a set of test functions $\left\{f_{\alpha}(t)\right\}$ and $\left\{g_{\alpha}(t)\right\}$. The Gaussian statistics implies

$$
\begin{aligned}
\ln G[f, g]= & -\int_{0}^{t} d \tau \int_{0}^{\tau} d s g_{\alpha}(\tau) \chi_{\alpha \beta}(\tau, s) f_{\beta}(s) \\
& -\int_{0}^{t} d \tau \int_{0}^{\tau} d s f_{\alpha}(\tau) \chi_{\alpha \beta}^{*}(\tau, s) g_{\beta}(s) \\
& -\int_{0}^{t} d \tau \int_{0}^{\tau} d s f_{\alpha}(\tau) \eta_{\alpha \beta}(\tau, s) f_{\beta}(s) \\
& -\int_{0}^{t} d \tau \int_{0}^{\tau} d s g_{\alpha}(\tau) \eta_{\alpha \beta}^{*}(\tau, s) g_{\beta}(s) .
\end{aligned}
$$

By functional derivation it follows that $\left\langle z_{\alpha}(t)\right\rangle=0$, and the correlations defined by Eqs. (3) and (4). These definitions cover the particular cases of real noises as well as the case where $\eta_{\alpha \beta}(t, s)=0$.

Novikov's theorem [37] gives an exact result for the mean value of the product between a Gaussian noise and any functional $\mathcal{M}$ of it. This theorem can be generalized 
for the set of complex noises defined by Eq. A.2. Using the Gaussian property and the correlation definition it is possible to obtain

$$
\begin{aligned}
\left\langle z_{\gamma}(t) \mathcal{M}\left[\left\{z_{\alpha}(t)\right\}\right]\right\rangle= & \int_{0}^{t} d s \chi_{\gamma \beta}^{*}(t, s)\left\langle\frac{\delta \mathcal{M}\left[\left\{z_{\alpha}(t)\right\}\right]}{\delta z_{\beta}^{*}(s)}\right\rangle(\mathrm{A} .3) \\
& +\int_{0}^{t} d s \eta_{\gamma \beta}(t, s)\left\langle\frac{\delta \mathcal{M}\left[\left\{z_{\alpha}(t)\right\}\right]}{\delta z_{\beta}(s)}\right\rangle
\end{aligned}
$$

where $\mathcal{M}\left[\left\{z_{\alpha}(t)\right\}\right]$ denotes a functional that depends on the set of noises $\left\{z_{\alpha}(t)\right\}$. The noise $z_{\gamma}(t)$ belongs to this set. This generalized Novikov theorem has been applied in the derivation of Sec. II.

\section{Correlation matrix}

The correlation matrix that define Eq. A.2 has to satisfy a positivity constraint. By defining the scalar $w \equiv$ $\sum_{i=1}^{n}\left(a_{i}^{\alpha} z_{\alpha}\left(t_{i}\right)+b_{i}^{\alpha} z_{\alpha}^{*}\left(t_{i}\right)\right)$, where $a_{i}^{\alpha}$ and $b_{i}^{\alpha}$ are arbitrary coefficients, $n \in \mathbf{N},\left\{t_{i}\right\}$ arbitrary times, the absolute value $|w|^{2} \geq 0$ implies that the four blocks correlation kernel

$$
\left(\begin{array}{ll}
\chi & \eta^{*} \\
\eta & \chi^{*}
\end{array}\right) \geq 0
$$

must be positive defined, where $\chi \leftrightarrow \chi_{\alpha \beta}\left(t_{i}, t_{j}\right)$ and $\eta \leftrightarrow \eta_{\alpha \beta}\left(t_{i}, t_{j}\right)$. An equivalent condition follows with $w \equiv \sum_{i=1}^{n}\left(a_{i}^{\alpha} \operatorname{Re}\left[z_{\alpha}\left(t_{i}\right)\right]+b_{i}^{\alpha} \operatorname{Im}\left[z_{\alpha}\left(t_{i}\right)\right]\right)$, leading to

$$
\left(\begin{array}{cc}
\operatorname{Re}[\chi+\eta] & \operatorname{Im}[\chi+\eta] \\
-\operatorname{Im}[\chi-\eta] & \operatorname{Re}[\chi-\eta]
\end{array}\right) \geq 0
$$

In both cases, when $n=1$ the positivity of the variance matrix of $\left\{z_{\alpha}(t)\right\}$ is recovered. Notice that $\eta_{\alpha \beta}(t, s)=$ $\chi_{\alpha \beta}(t, s)$ is an admissible correlation matrix only for real noises.

\section{a. Bosonic bath correlation matrix}

The correlation matrixes $\chi_{\alpha \beta}(t, s)$ and $\eta_{\alpha \beta}(t, s)$ corresponding to the diagonal map, Eq. (33) and (34) respectively, satisfy the positivity constraint (A.4). This property is fulfilled without imposing any special constraint on the bath properties or on the interaction Hamiltonian. In fact, by writing the interaction bath operators as linear combinations of free bosonic modes

$$
Z_{\alpha}(t)=\int d \omega\left(g_{\alpha \alpha^{\prime}}(\omega) b_{\alpha^{\prime} \omega}^{\dagger} e^{+i \omega t}+h_{\alpha \alpha^{\prime}}(\omega) b_{\alpha^{\prime} \omega} e^{-i \omega t}\right),
$$

with commutation relations $\left[b_{\alpha \omega}, b_{\beta \omega^{\prime}}^{\dagger}\right]=\delta_{\alpha \beta} \delta\left(\omega-\omega^{\prime}\right)$, it follows

$$
\begin{aligned}
\chi_{\alpha \beta}(t, s)= & \int d \omega\left(n_{\gamma \omega}+1\right) g_{\alpha \gamma}^{*}(\omega) g_{\beta \gamma}(\omega) e^{-i \omega(t-s)} \\
& +\int d \omega n_{\gamma \omega} h_{\alpha \gamma}^{*}(\omega) h_{\beta \gamma}(\omega) e^{+i \omega(t-s)},(\mathrm{A}
\end{aligned}
$$

where $n_{\gamma \omega}$ is the average thermal number in each mode. Given that $\eta_{\alpha \beta}(t, s)=0$, the positivity constraint (A.4) (at any bath temperature) is satisfied whenever the matrixes of complex coefficients $\left[\left(n_{\gamma \omega}+1\right) g_{\alpha \gamma}^{*}(\omega) g_{\beta \gamma}(\omega)\right]$ and $\left[n_{\gamma \omega} h_{\alpha \gamma}^{*}(\omega) h_{\beta \gamma}(\omega)\right]$ are positive defined, that is, the bath spectrum matrix is positive defined. On the other hand, we notice that non-stationary correlations 36] $\chi_{\alpha \beta}(t, s) \neq \chi_{\alpha \beta}(t-s)$, only arise if the underlying Hamiltonian (27) is time dependent. For example, the interaction strength may be time dependent, $\lambda \rightarrow \lambda \varphi(t), \chi_{\alpha \beta}(t, s) \rightarrow \varphi^{2}(t) \chi_{\alpha \beta}(t-s)$, or alternatively the previous interaction operators (A.5) are defined with time dependent coefficients $g_{\alpha \alpha^{\prime}}(\omega) \rightarrow g_{\alpha \alpha^{\prime}}(\omega, t)$, $h_{\alpha \alpha^{\prime}}(\omega) \rightarrow h_{\alpha \alpha^{\prime}}(\omega, t)$.

\section{b. Exponential noise correlations}

As an (one dimensional) example, we assume that the realizations of the noise $z(t)$ obey the linear stochastic differential equation

$$
\frac{d}{d t} z(t)=-(\gamma+i \Omega) z(t)+\xi(t),
$$

where $\gamma>0$ and $\Omega$ are real parameters. The complex Gaussian white noise $\xi(t)$ satisfies

$$
\begin{aligned}
\left\langle\xi^{*}(t) \xi(s)\right\rangle & =D \delta(t-s), \\
\langle\xi(t) \xi(s)\rangle & =D^{\prime} \delta(t-s),
\end{aligned}
$$

where $D^{\prime} \leq D$ are also real parameters. By integrating Eq. (A.7) as $z(t)=z(0) \exp [-(\gamma+i \Omega) t]+\int_{0}^{t} d t^{\prime} \exp [-(\gamma+$ $\left.i \Omega)\left(t-t^{\prime}\right)\right] \xi\left(t^{\prime}\right)$, and assuming stationary initial conditions for $z(t)$, it follows the exponential correlations

$$
\begin{aligned}
& \chi(t, s)=\left\langle z^{*}(t) z(s)\right\rangle=\frac{D}{2 \gamma} \exp [-(\gamma-i \Omega)(t-s)], \\
& \eta(t, s)=\langle z(t) z(s)\rangle=\frac{D^{\prime}}{2(\gamma+i \Omega)} \exp [-(\gamma+i \Omega)(t-s)],
\end{aligned}
$$

where $t \geq s$. In a Fourier domain, it is possible to demonstrate that these objects obey Eq. (A.4).

The degree of freedom introduced by $\eta(t, s)$ in Eq. (39) can be easily read by taking $\Omega=0$, leading to

$$
\chi(t, s)-\eta(t, s)=\left(1-\frac{D^{\prime}}{D}\right) \frac{D}{2 \gamma} \exp [-\gamma(t-s)] .
$$

Therefore, the dimensionless parameter $D^{\prime} / D$ allows to continuously departs from a Hamiltonian stochastic dynamics $\left(D^{\prime}=D\right)$ and, in the other extreme, to reach the standard non-Markovian diffusion model, that is, $D^{\prime}=0$. While this property was derived for this particular case, it is simple to realize that the inclusion of the extra correlations $\eta_{\alpha \beta}(t, s)$ allow to smoothly reach these limits when considering the general evolution (12) with an Hermitian operator base, $\left\{L_{\alpha}^{\dagger}\right\}=\left\{L_{\alpha}\right\}$. 
[1] H. P. Breuer and F. Petruccione, Theory of Open Quantum Systems (Oxford University Press, Oxford, England, 2002).

[2] A. Barchielli and M. Gregoratti, Quantum Trajectories and Measurements in Continuous time-The diffusive case, Lectures Notes in Physics Vol. 782 (Springer, Berlin, 2009).

[3] H. Wiseman and G. Milburn, Quantum Measurement and Control (Cambridge University Press, Cambridge, England, 2010).

[4] H. J. Carmichel, An Open Systems approach to Quantum Optics (Lectures Notes in Physics, Springer-Verlag, 1993).

[5] M. B. Plenio and P. L. Knight, The quantum jump approach to dissipative dynamics in quantum optics, Rev. Mod. Phys. 70, 101 (1998).

[6] N. Gisin and I. C. Percival, The quantum-state diffusion model applied to open systems, J. Phys. A: Math. Gen. 25, 5677 (1992); M. Rigo and N. Gisin, Unravellings of the master equation and the emergence of a classical world, Quantum Semiclass. Opt. 8, 255 (1996); N. Gisin and M. B. Cibils, Quantum diffusions, quantum dissipation and spin relaxation, J. Phys. A 25, 5165 (1992); L. Diósi, Quantum stochastic processes as models for state vector reduction, J. Phys. A 21, 2885 (1988).

[7] M. Rigo, F. Mota-Furtado, and P. F. O'Mahony, Continuous stochastic Schrödinger equations and localization, J. Phys. A: Math. Gen. 30, 7557 (1997).

[8] H. M. Wiseman and L. Diósi, Complete parametrization, and invariance, of diffusive quantum trajectories for Markovian open systems, Chem. Phys. 268, 91 (2001).

[9] A. Bassi and G. C. Ghirardi, Dynamical reduction models, Phys. Rep. 379, 257 (2003); N. Gisin and I. C. Percival, Quantum state diffusion, localization and quantum dispersion entropy, J. Phys. A: Math. Gen. 26, 2233 (1993); N. Gisin and I. C. Percival, The quantum state diffusion picture of physical process, J. Phys. A: Math. Gen. 26, 2245 (1993).

[10] L. Diósi and W. T. Strunz, The non-Markovian stochastic Schrödinger equation for open systems, Phys. Lett. A 235, 569 (1997); W. T. Strunz, Linear quantum state diffusion for non-Markovian open quantum systems, Phys. Lett. 224, 25 (1996); L. Diósi, Exact semiclassical wave equation for stochastic quantum optics, Quantum Semiclass. Opt. 8, 309 (1996).

[11] L. Diósi, N. Gisin, and W. T. Strunz, Non-Markovian quantum state diffusion, Phys. Rev. A 58, 1699 (1998).

[12] T. Yu, L. Diósi, N. Gisin and W. T. Strunz, NonMarkovian quantum-state diffusion: Perturbation approach, Phys. Rev. A 60, 91 (1999).

[13] J. D. Cresser, A Heisenberg Equation-of-motion Derivation of Stochastic Schrödinger Equations for NonMarkovian Open Systems, Laser Phys. 10, 1 (2000).

[14] A. A. Budini, Non-Markovian Gaussian stochastic wave vector, Phys. Rev. A 63, 012106 (2000).

[15] J. Gambetta and H. M. Wiseman, Perturbative approach to non-Markovian stochastic Schrödinger equations, Phys. Rev. A 66, 052105 (2002).

[16] Z. Li, C. Yip, H. Deng, M. Chen, T. Yu, J. Q. You, and C. Lam, Approach to solving spin-boson dynamics via non-Markovian quantum trajectories, Phys. Rev. A 90,
$022122(2014)$

[17] D. Suess, A. Eisfeld, and W. T. Strunz, Hierarchy of Stochastic Pure States for Open Quantum System Dynamics, Phys. Rev. Lett. 113, 150403 (2014).

[18] J. Xu, X. Zhao, J. Jing, L. Wu, and T. Yu, Perturbation methods for non-Markovian quantum state diffusion equation, J. Phys. A 47, 435301 (2014).

[19] W. T. Strunz, L. Diósi, and N. Gisin, Open System Dynamics with Non-Markovian Quantum Trajectories, Phys. Rev. Lett. 82, 1801 (1999).

[20] T. Yu, Non-Markovian quantum trajectories versus master equations: finite temperature heat bath, Phys. Rev. A 69, 062107 (2004).

[21] J. Jing and T. Yu, Non-Markovian Relaxation of a ThreeLevel System: Quantum Trajectory Approach, Phys. Rev. Lett. 105, 240403 (2010); J. Jing, X. Zhao, J. Q. You, and T. Yu, Time-local quantum-state-diffusion equation for multilevel quantum systems, Phys. Rev. A 85, 042106 (2012); Y. Chen, J. Q. You, and T. Yu, Exact non-Markovian master equations for multiple qubit systems: quantum trajectory approach, Phys. Rev. A 90, 052104 (2014).

[22] J. Jing and T. Yu, Stochastic Schrödinger equation for a non-Markovian dissipative Qubit-Qutrit system, Euro Phys. Lett. 96, 44001, (2011).

[23] L. Ferialdi and A. Bassi, Exact Solution for a NonMarkovian Dissipative Quantum Dynamics, Phys. Rev. Lett. 108, 170404 (2012).

[24] J. Jing, R. Li, J. Q. You, and T. Yu, Nonperturbative stochastic dynamics driven by strongly correlated colored noises, Phys. Rev. A 91, 022109 (2015).

[25] J. Gambetta and H. M. Wiseman, Non-Markovian stochastic Schrödinger equations: Generalization to realvalued noise using quantum-measurement theory, Phys. Rev. A 66, 012108 (2002).

[26] J. Gambetta and H. M. Wiseman, Interpretation of nonMarkovian stochastic Schrödinger equations as a hiddenvariable theory, Phys. Rev. A 68, 062104 (2003).

[27] L. Diósi, Non-Markovian Continuous Quantum Measurement of Retarded Observables, Phys. Rev. Lett. 100, 080401 (2008); H. M. Wiseman and J. M. Gambetta, Pure-State Quantum Trajectories for General Non-Markovian Systems Do Not Exist, Phys. Rev. Lett. 101, 140401 (2008).

[28] A. Bassi and G. C. Ghirardi, Dynamical reduction models with general Gaussian noises, Phys. Rev. A 65, 042114 (2002); A. Bassi, Stochastic Schrödinger equations with general complex Gaussian noises, Phys. Rev. A 67, 062101 (2003); S. Adler and A. Bassi, Collapse models with non-white noises, J. Phys. A 40, 15083 (2007); A. Bassi and L. Ferialdi, Non-Markovian dynamics for a free quantum particle subject to spontaneous collapse in space: general solution and main properties, Phys. Rev. A 80, 012116 (2009); L. Ferialdi and A. Bassi, Dissipative collapse models with non-white noises, Phys. Rev. A 86, 022108 (2012).

[29] A. Bassi and L. Ferialdi, Non-Markovian Quantum Trajectories: An Exact Result, Phys. Rev. Lett. 103, 050403 (2009).

[30] D. Alonso and I. de Vega, Multiple-time Correlation Function For Non-Markovian Interaction: Beyond The 
Quantum Regression Theorem, Phys. Rev. Lett. 94, 200403 (2005); D. Alonso and I. de Vega, Hierarchy of equations of multiple-time correlation functions, Phys. Rev. A 75, 052108 (2007).

[31] W. T. Strunz, L. Diósi, N. Gisin, and T. Yu, Quantum Trajectories for Brownian Motion, Phys. Rev. Lett. 83, 4909 (1999); W. T. Strunz and T. Yu, Convolutionless Non-Markovian master equations and quantum trajectories: Brownian motion revisited, Phys. Rev. A 69, 052115 (2004).

[32] J. Roden, W. T. Strunz, and A. Eisfeld, Non-Markovian quantum state diffusion for absorption spectra of molecular aggregates, J. Chem. Phys. 134, 034902 (2011); J. Roden, A. Eisfeld, W. Wolff, and W. T. Strunz, Influence of Complex Exciton-Phonon Coupling on Optical Absorption and energy Transfer of Quantum Aggregates, Phys. Rev. Lett. 103, 058301 (2009).

[33] G. Ritschel, D. Suess, S. Möbius, W. T. Strunz, and A. Eisfeld, Non-Markovian Quantum State Diffusion for temperature-dependent linear spectra of light harvesting aggregates, J. Chem. Phys. 142, 034115 (2015).

[34] X. Zhong and Y. Zhao, Non-Markovian stochastic Schrödinger equation at finite temperatures for charge carrier dynamics in organic crystals, J. Chem. Phys. 138, 014111 (2013).

[35] J. Jing, X. Zhao, J. Q. You, W. T. Strunz, and T. Yu, Many-body quantum trajectories of non-Markovian open systems, Phys. Rev. A 88, 052122 (2013).

[36] L. Diósi and L. Ferialdi, General Non-Markovian Structure of Gaussian Master and Stochastic Schrödinger Equations, Phys. Rev. Lett. 113, 200403 (2014).

[37] A. Novikov, Functionals and the random-force method in turbulence theory, Soviet Phys. JETP 20, 1290 (1965).

[38] A. A. Budini, Quantum systems subject to the action of classical stochastic fields, Phys. Rev. A 64, 052110 (2001). 